\title{
Solitons on the edge of a two-dimensional electron system
}

\author{
C. Wexler and Alan T. Dorsey \\ Department of Physics, University of Florida, Gainesville, Florida 32611-8440
}

(1 Oct 1998, revised 5 Dec 1998)

\begin{abstract}
We present a study of the excitations of the edge of a two-dimensional electron droplet in a magnetic field in terms of a contour dynamics formalism. We find that, beyond the usual linear approximation, the non-linear analysis yields soliton solutions which correspond to uniformly rotating shapes. These modes are found from a perturbative treatment of a non-linear eigenvalue problem, and as solutions to a modified Korteweg-de Vries equation resulting from a local induction approximation to the nonlocal contour dynamics. We discuss applications to the edge modes in the quantum Hall effect.
\end{abstract}

PACS numbers: 73.40.Hm, 02.40.Ma, 03.40.Gc, 11.10.Lm

Shape deformations are important for our understanding of such diverse problems as the low-lying excitations of atomic nuclei [1], the hydrodynamics of vortex patches [2,3], the evolution of atmospheric plasma clouds [1], the formation of patterns in magnetic fluids and superconductors [5], and the dynamics of suspended liquid droplets [6,7], to name just a few examples. In many of these systems there is a field which is approximately piecewise constant (for example, the vorticity for the vortex patches, or the magnetization for the magnetic fluids); this greatly simplifies the study of the dynamics, since it is often possible to focus attention on the boundaries at which this field is discontinuous, and construct the contour dynamics for these boundaries, subject to some global constraint (such as area conservation).

The edges of a two-dimensional electron system (2DES), and in particular the edges of a quantum Hall (QH) liquid, present a unique opportunity to study the dynamics of shape deformations in a clean and controlled environment. The 2DES in the QH state is incompressible, so that the electron density is approximately piecewise constant, suggesting that a contour dynamics approach to studying the droplet excitations is viable. In addition, the charged nature of the system facilitates the excitation and detection of deformations of the droplet.

In this paper we will formulate the study of the excitations of a droplet in a 2DES as a problem in contour dynamics. In the usual treatment of the edge excitations [8], a linearization of the equation of motion is done at early stages, thus limiting the applicability to small deformations of the edge of the system from an unperturbed state. In this Letter we consider non-linear terms which are present in the full contour dynamics treatment. We first present perturbative results for non-linear deformations of the 2DES shape. We then show that the local induction approximation to the full contour dynamics generates the modified Korteweg-de Vries (mKdV) [9] equation for the curvature dynamics; the $\mathrm{mKdV}$ equation also arises in studies of vortex patches [3] and suspended liquid droplets [7]. The $\mathrm{mKdV}$ dynamics conserve an infinite number of quantities, including the area, center of mass, and angular momentum of the droplet 10, 11], so that our local approximation to the nonlocal dynamics preserves the important conservation laws. The $\mathrm{mKdV}$ equation also possesses soliton solutions, including traveling wave solutions which represent uniformly rotating deformed droplets.

I. Hydrodynamics of a two-dimensional electron system in a magnetic field.-Consider a 2DES in a strong magnetic field. The electron configuration can, in most cases, be characterized by its density $n(\mathbf{r})$ and its velocity $\mathbf{v}(\mathbf{r})$. Treated as a classical fluid, the dynamics is determined by the Euler and continuity equations,

$$
\begin{gathered}
\dot{\mathbf{v}}=-\omega_{c} \mathbf{e}_{z} \times \mathbf{v}-\frac{e^{2}}{m_{e} \epsilon} \nabla \int d^{2} r^{\prime} \frac{n\left(\mathbf{r}^{\prime}\right)}{\left|\mathbf{r}-\mathbf{r}^{\prime}\right|}+\frac{e}{m_{e}} \mathbf{E}_{\mathrm{ext}}, \\
\partial_{t} n+\boldsymbol{\nabla} \cdot(n \mathbf{v})=0
\end{gathered}
$$

where $\omega_{c}=e B / m_{e}$ is the cyclotron frequency and $\epsilon$ is the dielectric constant of the medium; the first term on the right hand side of Eq. (11) is the Lorentz force, the second is the Coulomb interaction, and $\mathbf{E}_{\text {ext }}$ is the electric field due to the background positive charge, gates, etc. It is noteworthy that this simple hydrodynamic theory of edge deformations, and the corresponding canonical quantization of the classical Lagrangian of the fluid, is also able to capture the essence of the many-body problem in both integer and fractional QH states [8].

The theory of small deformations of the edge has been extensively studied in Refs. 12 14. The main conclusion is that for strong magnetic fields, when Landau level quantization becomes important, the only low energy modes are edge modes which propagate in one direction along the edge of the 2DES; the bulk modes become gaped with a minimum frequency $\omega_{c}$. For a sharp electron density profile, this edge mode is the "conventional" edge magnetoplasmon mode, with a dispersion relation:

$$
\omega_{0}(k)=-2 \ln \left(\frac{e^{-\gamma}}{2|k a|}\right) \frac{\bar{n} e^{2}}{\epsilon m_{e} \omega_{c}} k,
$$

where $k$ is the mode wave-number, $\gamma \approx 0.5772$ is the Euler constant, and $a$ is a short-distance cut-off [12] (the 
largest of the transverse width of the 2DES, the magnetic length, or the width of the compressible edge-channel). In addition, for the more realistic wide and compressible edges, new branches of "acoustic" modes exist [14]. Recent time-of-flight measurements [15] on 2DES in heterostructures confirm this simple picture.

II. Edge modes: dynamics and kinematics.-For what follows, let us consider the simplest case: a sharp edge between an incompressible charged fluid and "vacuum" (Fig. 1). We focus on this mode since it has the simplest structure and is the most readily observable [12,15]. As in Ref. [12], we neglect inertial terms on the left hand side of Eq. (1), and find that the electron velocity is given by

$$
\mathbf{v}(\mathbf{r})=-\frac{e^{2}}{\epsilon m_{e} \omega_{c}} \nabla \times \mathbf{e}_{z} \int_{\mathcal{A}} d^{2} r^{\prime} \frac{n\left(\mathbf{r}^{\prime}\right)}{\left|\mathbf{r}-\mathbf{r}^{\prime}\right|}+\mathbf{v}_{\mathrm{ext}}
$$

where $\mathbf{v}_{\text {ext }}=-\left(e / m_{e} \omega_{c}\right) \mathbf{e}_{z} \times \mathbf{E}_{\text {ext }}$ is the velocity induced by the external field, and $\mathcal{A}$ is the area of the droplet.

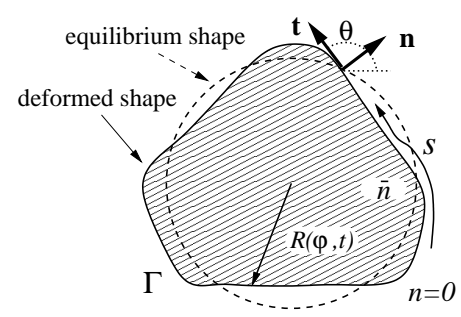

FIG. 1. A charged incompressible liquid in a magnetic field. We assume a piecewise constant electron density $(n=\bar{n}$ inside, while $n=0$ outside the droplet). The parameterization $R(\varphi, t)$, the tangent $\mathbf{t}$ and normal $\mathbf{n}$ unit vectors to the boundary $\Gamma$ are indicated; $s$ is the arclength and $\theta$ the tangent angle.

Let us now concentrate on the "internal" velocity given by the first term in Eq. (4) [16]. For an incompressible 2DES with a piecewise constant density [17, the density can be taken outside the integral; then using Stokes' theorem, the area integral can be transformed into a line integral over the boundary $\Gamma=\partial \mathcal{A}$ of the electron liquid:

$$
\mathbf{v}(\mathbf{r})=\frac{\bar{n} e^{2}}{\epsilon m_{e} \omega_{c}} \oint_{\Gamma} d s^{\prime} \frac{\mathbf{t}\left(s^{\prime}\right)}{\left|\mathbf{r}-\mathbf{r}\left(s^{\prime}\right)\right|} .
$$

Here $\mathbf{t}\left(s^{\prime}\right)=\partial_{s^{\prime}} \mathbf{r}\left(s^{\prime}\right) \equiv \mathbf{r}_{s^{\prime}}$ is the unit tangent vector at the arc-length $s^{\prime}$; for later use, we also define $\mathbf{n} \perp \mathbf{t}$ as the unit normal vector. The short distance singularity in the integrand is cut off at a length scale $r_{0}$. Equation (5) forms the basis of our contour dynamics treatment-it expresses the velocity of the edge in terms of a nonlocal self-interaction of the edge.

For the sake of comparison, we draw analogy to the case of a vortex patch, a two-dimensional, bounded region of constant vorticity $\omega_{p}$ surrounded by an irrotational fluid, where the interaction is logarithmic [2,3]:

$$
\mathbf{v}(\mathbf{r})=-\frac{\omega_{p}}{2 \pi} \oint_{\Gamma} d s^{\prime} \mathbf{t}\left(s^{\prime}\right) \ln \left[\frac{\left|\mathbf{r}-\mathbf{r}\left(s^{\prime}\right)\right|}{r_{0}}\right] .
$$

The vorticity and the area of the patch are conserved, a consequence of Kelvin's circulation theorem [18]. We see that in both cases (i) the dynamics is chiral, being determined by the tangent vector; (ii) the fluid contained within $\Gamma$ is incompressible, so that the area is conserved. It is this analogy which inspired the present work.

Having determined the velocity of the electron liquid, we now focus on the motion of the 2DES boundary $\Gamma$. The velocity of a point on the boundary can be written in terms of the normal and tangential components,

$$
\mathbf{v}=U(s) \mathbf{n}+W(s) \mathbf{t} .
$$

The tangential velocity $W(s)$ is largely irrelevant. We now ask whether there are modes which propagate along the boundary with no change in shape. Previous work 12] has focused on small perturbations of a straight, infinite edge. Here we consider deformations of a circular droplet of incompressible electrons. A uniformly propagating mode is therefore characterized by a boundary that moves like a rotating rigid body, namely the radius of the boundary satisfies $R(\varphi, t)=R(\varphi-\Omega t)$, where $\varphi$ is the azimuthal angle and $\Omega$ is the angular frequency. This translates into a condition for the normal velocity:

$$
\left.U \equiv \mathbf{n}(\mathbf{r}) \cdot \mathbf{v}(\mathbf{r})\right|_{\mathbf{r} \in \Gamma}=\Omega \mathbf{n}(\mathbf{r}) \cdot\left(\mathbf{e}_{z} \times \mathbf{r}\right)
$$

We seek boundary shapes $R(\varphi)$ (see Fig. 1) that rotate uniformly, satisfying Eq. (8). Consider the following parameterization of the surface:

$$
R(\varphi)=R_{0}\left(1+\sum_{l=-\infty}^{\infty} b_{l} e^{i l \varphi}\right)
$$

The unit tangent vector is given by $\mathbf{t}(\varphi)=\boldsymbol{\tau}(\varphi) /|\boldsymbol{\tau}(\varphi)|$ where $\boldsymbol{\tau}(\varphi) \equiv \partial \mathbf{r}(\varphi) / \partial \varphi=\mathbf{e}_{r} R^{\prime}(\varphi)+\mathbf{e}_{\varphi} R(\varphi)$. Likewise, the unit normal vector is given by $\mathbf{n}(\varphi)=-\mathbf{e}_{z} \times \boldsymbol{\tau}(\varphi) /|\boldsymbol{\tau}(\varphi)|$. The normal velocity on the boundary can be written as

$$
U(\varphi)=\frac{\bar{n} e^{2}}{\epsilon m_{e} \omega_{c}} \int_{0}^{2 \pi} d \varphi^{\prime} \frac{\mathbf{n}(\varphi) \cdot \boldsymbol{\tau}\left(\varphi^{\prime}\right)}{\left|\mathbf{R}(\varphi)-\mathbf{R}\left(\varphi^{\prime}\right)\right|} .
$$

It has not been possible to solve exactly the non-linear eigenvalue problem for $b_{l}$ and $\Omega$ [Eqs. (8-10)] exactly. We therefore seek a perturbative solution by expanding the right-hand side of Eq. (10) in powers of $b_{l}$. This allows us to to go beyond the linear approximations used in the past, and we have succeeded in calculating shape deformations to $\mathcal{O}\left[b_{l}^{4}\right]$ and angular frequencies to $\mathcal{O}\left[b_{l}^{5}\right]$. Expanding the non-linear eigenvalue problem to fifth order, we find the condition

$$
\begin{aligned}
& \tilde{\Omega}\left(b_{l}+\frac{1}{2} \sum_{p} b_{l-p} b_{p}\right)=Q_{l} b_{l}+\sum_{p} R_{l, p} b_{l-p} b_{p} \\
& \quad+\sum_{p, q} S_{l, p, q} b_{l-p} b_{p-q} b_{q}+\sum_{p, q, r} T_{l, p, q, r} b_{l-p} b_{p-q} b_{q-r} b_{r} \\
& \quad+\sum_{p, q, r, s} U_{l, p, q, r} b_{l-p} b_{p-q} b_{q-r} b_{r-s} b_{s}+\mathcal{O}\left[b_{l}^{6}\right]
\end{aligned}
$$


where $\tilde{\Omega}=\left(\epsilon m_{e} \omega_{c} R_{0} / \bar{n} e^{2}\right) \Omega$, and the "matrix elements" $Q, R, S, T$ and $U$ are obtained from Eq. (10). The details of this calculation will be published elsewhere [10]. The zeroth order angular frequency for the eigenmode with rotational symmetry $C_{l}$ (rotations by $2 \pi / l$ ) is

$$
\tilde{\Omega}^{(0)}[l]=Q_{l}=4 \sum_{k=2}^{|l|} \frac{1}{2 k-1}
$$

where the last sum can be related to the digamma function $\psi(|l|+1 / 2)$. This linear result has been previously derived by several authors 13 ; corrections are $\mathcal{O}\left[b_{l}^{2}\right]$. For a direct comparison with Eq. (3), which corresponds to the large-l limit, we substitute the asymptotic expansion for the sum in Eq. (12); multiplication by $R_{0}$ yields the propagation velocity:

$$
v_{g}=-2 \ln \left(\frac{e^{-\gamma}}{4 e^{2}|l|}\right) \frac{\bar{n} e^{2}}{\epsilon m_{e} \omega_{c}},
$$

which closely corresponds to $v_{g} \equiv \partial \omega_{0}(k) / \partial k$ after the substitution $l \sim k a$ [see Eq. (3)]. The dispersion for these linear edge excitations has been confirmed experimentally in both the frequency [19] and time [15,20] domains.

Some of the shapes are shown by the dotted curves in Fig. 2, and closely resemble the "V states" of vortex patches found by Deem and Zabusky [21]. For larger deformations, the appearance of oscillations indicate that higher order terms are needed, since they correspond to higher Fourier components.

III. Local induction approximation.-As we have seen, the motion of the edge is determined by the velocity of the fluid at the surface. The nonlocal equation for the velocity of the boundary, Eq. (5), can be turned into a differential equation for the curvature of the boundary if we concentrate on the local contributions. This $l o-$ cal induction approximation (LIA) [18] was explored by Goldstein and Petrich in a series of papers dealing with the evolution of vortex patches [3,11]. The situation is considerably more favorable in this problem, due to the more rapid decay of the interaction $[1 / r$ for charges vs. $\ln (r)$ for vortices, see Eqs. (5) and (6)].

The LIA is an expansion of the integrand of Eq. (5) about $s^{\prime}=s$; a long-distance cut-off $\Lambda$ is introduced by replacing the line integral $\oint_{\Gamma}\{\cdots\} d s^{\prime}$ by $\int_{s-\Lambda / 2}^{s+\Lambda / 2}\{\cdots\} d s^{\prime}$. Using the Frenet-Serret relations $\mathbf{r}_{s}=\mathbf{t}, \mathbf{r}_{s s}=\mathbf{t}_{s}=-\kappa \mathbf{n}$, where $\kappa=\theta_{s}$ is the local curvature of the boundary, to lowest order the normal and tangential velocities are [10]:

$$
\begin{aligned}
U_{\text {LIA }} & =-\left[\frac{\bar{n} e^{2}}{\epsilon m_{e} \omega_{c}}\right] \frac{\Lambda^{2}}{8} \kappa_{s}, \\
W_{\text {LIA }} & =\left[\frac{\bar{n} e^{2}}{\epsilon m_{e} \omega_{c}}\right]\left(\ln \frac{\Lambda^{2}}{2 r_{0}}-\frac{11 \Lambda^{2}}{96} \kappa^{2}\right) .
\end{aligned}
$$

Since the rate of change of the area of the droplet is $\mathcal{A}_{t}=\oint d s U(s)$, the LIA, with $U_{\text {LIA }} \propto \kappa_{s}$, automatically conserves area.
The time evolution of a curve in two dimensions is given quite generally by the differential equation [11,22

$$
\kappa_{t}=-\left[\kappa^{2}+\partial_{s s}\right] U+\kappa_{s} W-\kappa_{s} \int_{0}^{s}\left[\kappa U+W_{s^{\prime}}\right] d s^{\prime} .
$$

Introducing the results from Eq. 14, changing "gauge" (by modifying $W$ ) and rescaling time, we find that the curvature satisfies the mKdV equation [9]:

$$
\kappa_{t}=\frac{3}{2} \kappa^{2} \kappa_{s}+\kappa_{s s s} .
$$

The mKdV dynamics are integrable, with an infinite number of globally conserved geometric quantities [3], the most important of which are the center of mass, area, and angular momentum of the droplet.

The mKdV equation possesses a variety of soliton solutions, including traveling wave solutions and propagating "breather" solitons. Here we will focus on the traveling wave solutions of Eq. (16) of the form $\kappa(s, t)=g(z)$ with $z \equiv s-c t$, which represent uniformly rotating deformed droplets. The ordinary differential equation for $g(z)$ can be integrated twice with the result

$$
\frac{1}{2}\left(g^{\prime}\right)^{2}=-\frac{1}{8} g^{4}+\frac{1}{2} c g^{2}+a g-2 b,
$$

where $a$ and $b$ are constants of integration $(a=b=0$ for infinite systems). The periodic solutions of this equation, expressed in terms of Jacobi elliptic functions, are:

$$
\begin{aligned}
\kappa(z) & =\frac{\left(q \kappa_{\max }+p \kappa_{\min }\right)+\left(p \kappa_{\min }-q \kappa_{\max }\right) \operatorname{cn}\left(\sqrt{p q} \frac{z}{2} \mid \lambda\right)}{(p+q)+(p-q) \operatorname{cn}\left(\sqrt{p q} \frac{z}{2} \mid \lambda\right)}, \\
p^{2} & =\left(3 \kappa_{\max }+\kappa_{\min }\right)^{2}+\xi^{2} \\
q^{2} & =\left(\kappa_{\max }+3 \kappa_{\min }\right)^{2}+\xi^{2} \\
\lambda^{2} & =\left[\left(\kappa_{\max }-\kappa_{\min }\right)^{2}-(p-q)^{2}\right] / 4 p q,
\end{aligned}
$$

where $\kappa_{\max \text { min }}$ are the maximum and minimum curvatures, and $\xi$ is determined by the boundary conditions. The period of $\kappa$ is given by the elliptic integral $L_{\kappa} \equiv(8 / \sqrt{p q}) K(\lambda)$. The first integral of the curvature gives the tangent angle $\theta(s)=\int_{0}^{s} \kappa\left(s^{\prime}\right) d s^{\prime}$. We require that $\theta\left(l L_{\kappa}\right)=2 \pi$, so that the resulting curve is closed (this fixes $\xi$ ). The integer $l$ determines that the curve has $C_{l}$ symmetry. The curves thus generated can be characterized by $\left(l, \kappa_{\max }, \kappa_{\min }\right)$ or more conveniently by the symmetry, the area and the perimeter of the curve.

The full lines in Fig. 2 show some uniformly rotating soliton shapes, calculated from Eq. (18). These are essentially identical with Goldstein and Petrich's 11] soliton solutions for the vortex patch problem (Deem and Zabusky's "V states" 21]), but the more local interaction in the QH case guarantees a better correspondence with the perturbative solutions. Indeed, the curves resulting from the perturbative method and the LIA are quite close, even for considerable deformations of the 
boundary. For larger deformations the perturbative results show artifacts due to the limited number of Fourier components. The advantage of the LIA becomes evident in this case, since it is an expansion in powers of the curvature and not the deformation, and thus allows for relatively large long-wavelength deformations. More significantly, the LIA and the resulting integrable dynamics allow one to uncover geometrical conservation laws which would be hidden in a perturbative calculation [10]. This advantage comes at a price: the detailed information on frequencies is obscured by the introduction of the long distance cut-off $\Lambda$ and by the gauge transformation of the tangential velocity $W$, while the frequency is easily obtained in the perturbative calculation.

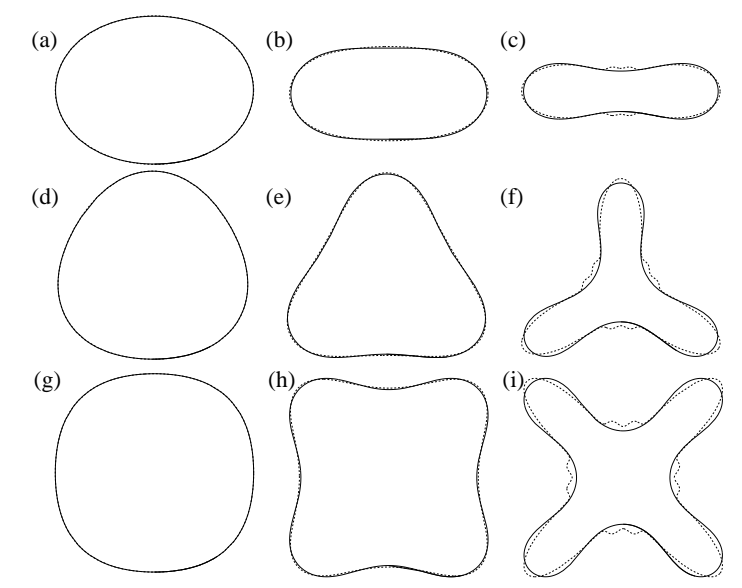

FIG. 2. Uniformly rotating shapes of a 2DES. Solid lines: solutions of the $\mathrm{mKdV}$ equation obtained from the local induction approximation. Dotted lines: solutions obtained using the perturbative expansion. The values of the coefficient $b_{l}$, and the ratio of curvatures $\sigma \equiv \kappa_{\min } / \kappa_{\max }$ are: (a) $b_{2}=0.073, \sigma=0.4$; (b) $b_{2}=0.19, \sigma=0$; (c) $b_{2}=0.36, \sigma=-0.2$; (d) $b_{3}=0.027, \sigma=0.4 ;$ (e) $b_{3}=0.10, \sigma=-0.2 ;$ (f) $b_{3}=0.29, \sigma=-0.45 ; \quad(\mathrm{g}) b_{4}=0.014, \sigma=0.4 ; \quad(\mathrm{h})$ $b_{4}=0.089, \sigma=-0.4$; (i) $b_{4}=0.24, \sigma=-0.56$.

$I V$. Conclusions.-A contour dynamics formulation of the excitations on the edge of a two-dimensional electron system in a magnetic field has allowed us to demonstrate the existence, beyond the usual linear regime, of shape deformations that propagate uniformly. A local approximation to the nonlocal dynamics shows that the curvature of the edge of the droplet obeys the modified Korteweg-de Vries equation, which has integrable dynamics and soliton solutions. Since these solutions are dispersionless, it may be possible to distinguish them from linear edge waves in time-of-flight measurements. Earlier studies 23] have shown non-linear waves in edge channels, but the origin of the non-linearity and experimental details are different than considered here. On the theoretical side, it would be interesting to connect our hydrodynamic treatment of these edge solitons with field-theoretical treatments of edge excitations [13].
We would like to thank Raymond Goldstein for useful discussions. This work was supported by the NSF grant DMR-9628926.

[1] A. Bohr, Mat. Fys. Medd. Dan. Vid. Selsk. 26 (1952) no. 14.

[2] N. J. Zabusky et al., J. Comp. Phys. 30, 96 (1979); N. J. Zabusky and E. A. Overman, ibid 52, 351 (1983).

[3] R. E. Goldstein and D. M. Petrich, Phys. Rev. Lett. 69, 555 (1992).

[4] E. A. Overman and N. J. Zabusky, Phys. Rev. Lett. 45, 1693 (1980).

[5] R. E. Rosensweig, Ferrohydrodynamics (Cambridge Univ. Press, 1985); S. A. Langer, R. E. Goldstein and D. P. Jackson, Phys. Rev. A 46, 4894 (1992); A. T. Dorsey and R. E. Goldstein, Phys. Rev. B 57, 3058 (1998).

[6] R. G. Holt and E. H. Trinh, Phys. Rev. Lett. 77, 1274 (1996); R. E. Apfel et al., Phys. Rev. Lett. 78, 1912 (1997)

[7] A. Ludu and J. P. Draayer, Phys. Rev. Lett. 80, 2125 (1998).

[8] See X. G. Wen, Int. J. Mod. Phys. B 6, 1711 (1992); and C. L. Kane and M. P. A. Fisher, in Perspectives in Quantum Hall Effects, edited by S. Das Sarma and A. Pinczuk (Wiley, New York, 1997), pp. 109-159.

[9] D. J. Korteweg and G. de Vries, Phil. Mag. (5), 39, 422 (1895); see A. Das, Integrable Models (World Scientific, Singapore, 1989).

[10] C. Wexler and A. T. Dorsey, in preparation.

[11] R. E. Goldstein and D. M. Petrich, Phys. Rev. Lett. 67, 3203 (1991); in Singularities in Fluids, Plasmas and Optics (Kluwer Acad., 1993), pp. 93-109.

[12] V. A. Volkov and S. A. Mikhailov, Zh. Eksp. Teor. Fiz. 94217 (1988) [Sov. Phys. JETP 67, 1639 (1988)].

[13] S. Giovanazzi et al., Phys. Rev. Lett. 72, 3230 (1994); A. Cappelli et al., Ann. Phys. 246, 86 (1996).

[14] I. L. Aleiner and L. I. Glazman, Phys. Rev. Lett. 72, 2935 (1994).

[15] G. Ernst et al., Phys. Rev. Lett. 77, 4245 (1996).

[16] The external field is important for long term stability, and also modifies the propagation velocity. In general it will also change the shape of the modes, yet one can devise situations in which these effects are not important, for instance in a parabolic confining potential.

[17] In general, the electron density increases from zero to its bulk value over a certain distance $a$. Our approximation should be valid for deformations with characteristic length scales much larger than $a$.

[18] See G. K. Batchelor, An Introduction to Fluid Dynamics (Cambridge University Press, Cambridge, 1967), Ch. 7.

[19] S. J. Allen et al., Phys. Rev. B 28, 4875 (1983); M. Wassermeier et al., Phys. Rev. B 41, 10287 (1990); I. Grodnesky et al., Phys. Rev. Lett. 67, 1019 (1991).

[20] R. C. Ashoori et al., Phys. Rev. B 45, 3894 (1992). G. Ernst et al., Phys. Rev. Lett. 79, 3748 (1997).

[21] G. S. Deem and N. J. Zabusky, Phys. Rev. Lett. 40, 859 (1978).

[22] R. C. Brower et al., Phys. Rev. A 29, 1335 (1984).

[23] N. B. Zhitenev et al., Phys Rev. B 52, 11277 (1995). 